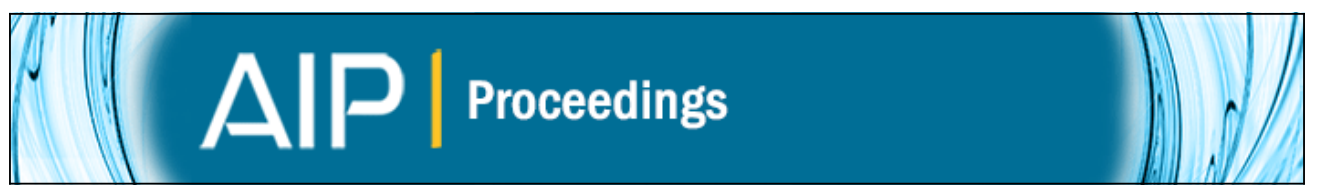

\title{
Assembly of the First Dwarf Galaxies
}

Andreas Pawlik, Miloš Milosavljević, and Volker Bromm

Citation: AIP Conference Proceedings 1294, 272 (2010); doi: 10.1063/1.3518873

View online: http://dx.doi.org/10.1063/1.3518873

View Table of Contents:

http://scitation.aip.org/content/aip/proceeding/aipcp/1294?ver=pdfcov

Published by the AIP Publishing

\section{Articles you may be interested in}

Assembly of the first disk galaxies under radiative feedback from pop III stars

AIP Conf. Proc. 1480, 234 (2012); 10.1063/1.4754360

The imprint of pop III stars on the first galaxies

AIP Conf. Proc. 1480, 123 (2012); 10.1063/1.4754341

Observational Signatures of the First Galaxies

AIP Conf. Proc. 1294, 190 (2010); 10.1063/1.3518851

The Origin of the Diversity of Dwarf Spheroidal Galaxies

AIP Conf. Proc. 1240, 421 (2010); 10.1063/1.3458554

Towards the First Galaxies

AIP Conf. Proc. 990, 405 (2008); 10.1063/1.2905651 


\title{
Assembly of the First Dwarf Galaxies
}

\author{
Andreas Pawlik, Miloš Milosavljević and Volker Bromm \\ Department of Astronomy and Texas Cosmology Center, \\ The University of Texas at Austin, TX 78712
}

\begin{abstract}
Understanding the formation and evolution of the first stars and galaxies is crucial to understanding reionization, a key epoch in the history of the Universe. Detailed theoretical studies of the galaxies before and during reionization are now particularly urgent because of the wealth of observational data that will soon be provided by the next generation of telescopes, such as JWST, ALMA, LOFAR, MWA, and others. We simulate the formation of the first galaxies using cosmological smoothed particle hydrodynamics simulations. Zooming in on individual galaxies, we explore how various physical processes affect their assembly and further evolution. A highlight of our study will be the simulation of the radiation-hydrodynamics of galaxy assembly, which we will perform using our multi-frequency radiative transfer method TRAPHIC. Feedback from radiation has long been suspected to play a decisive role in galaxy formation and we will investigate its implications for observable properties of the first galaxies.
\end{abstract}

Keywords: galaxies: formation - galaxies: high-redshift - hydrodynamics - radiative transfer PACS: 95.30.Lz; 97.10.Bt; 97.20.Wt; 95.30.Jx; 98.62.Ai

\section{NUMERICAL METHOD}

We use a modified version of the N-body Tree-PM smoothed particle hydrodynamics code P-Gadget3-BG [1, 2]. Starting from WMAP5 [3] LCDM cosmological initial conditions, we perform zoomed simulations of galaxies in halos reaching masses of $\sim 10^{9} \mathrm{M}_{\odot}$ at redshift $z \approx 10$. We follow the non-equilibrium $\mathrm{H} 2+\mathrm{HD}$ chemistry and cooling of primordial gas. Star formation is implemented by turning gas particles into star particles as described in [4].

To study radiative feedback, we use our multi-frequency radiative transfer method TRAPHIC ([5]), which solves the time-dependent radiative transfer problem in primordial gas $(\mathrm{H}+\mathrm{He})$. TRAPHIC's distinguishing features include that it is spatially adaptive, parallel on distributed memory and has a computation time that does not scale with the number of sources. Hence it is ideal for application in spatially adaptive cosmological simulations that contain a large number of ionizing sources. The latest version of TRAPHIC is implemented in P-Gadget3-BG and is fully radiation-hydrodynamically coupled (to be described elsewhere).

\section{INITIAL RESULTS}

Figure 1 shows the gas densities at $z=10$ at two different scales in one of our zoomed simulations. The simulation employed a gas mass resolution of $484 \mathrm{M}_{\odot}$ and a gravitational softening of (Plummer-equivalent) radius $0.1 h^{-1} \mathrm{kpc}$ comoving. It included atomic cooling and was performed without radiative transfer. Hence, it will provide a 

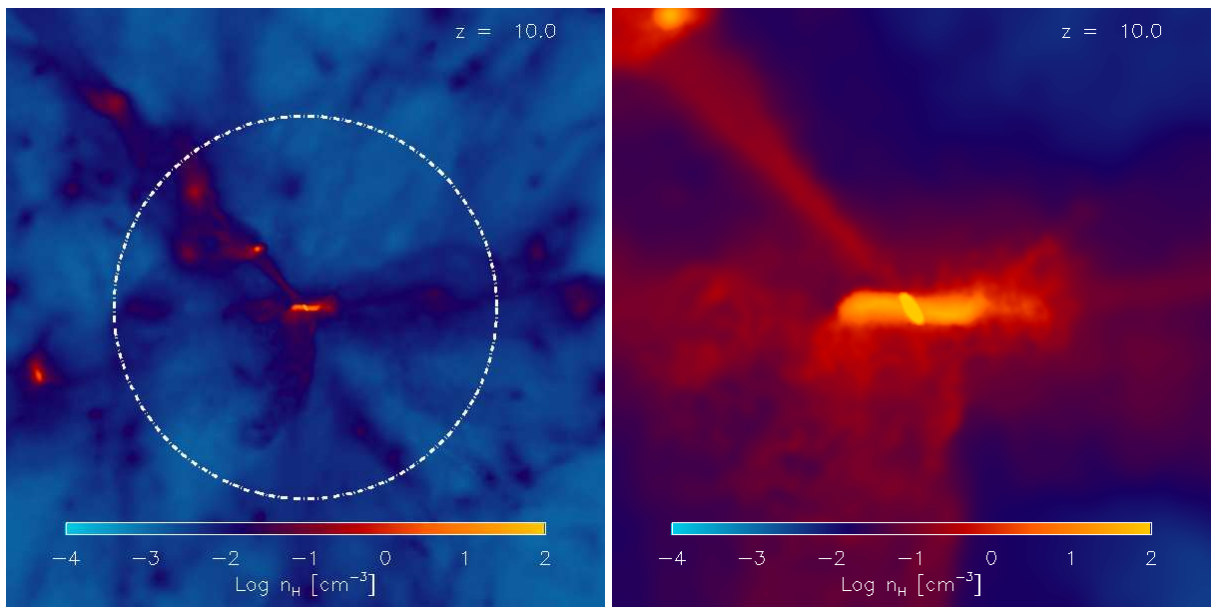

FIGURE 1. Hydrogen number densities at $z=10$ in the no-feedback simulation. The circle marks the virial region with physical radius $r_{\mathrm{vir}} \approx 3 \mathrm{kpc}$. The right-hand image is a zoom-in on the virial region.

useful reference for comparison to simulations that include the transfer of radiation and associated feedback. The inner and outer disks that characterize the morphology of the galaxy were assembled after mergers at redshifts $z \approx 15$ and $z \approx 12$.

\section{OUTLOOK}

Simulating the first galaxies is a demanding task which requires non-equilibrium chemistry, primordial gascooling, ionizing photon transport and feedback from star formation in high-resolution cosmological runs. The results presented here are the first step towards first-principle predictions for the first galaxies that can soon be tested with the James Webb Space Telescope and other future observatories.

\section{ACKNOWLEDGMENTS}

This research is supported by the NASA grant NNX09AJ33G. A. H. P. thanks Joop Schaye, Claudio Dalla Vecchia and Freeke van de Voort for useful discussions.

\section{REFERENCES}

1. Schaye J., et al., 2010, MNRAS, 402, 1536

2. Springel V., 2005, MNRAS, 364, 1105

3. Komatsu E., et al., 2009, ApJS, 180, 330

4. Schaye J., Dalla Vecchia C., 2008, MNRAS, 383, 1210

5. Pawlik A. H., Schaye J., 2008, MNRAS, 389, 651 